\title{
mRNA Quantification of NIPBL Isoforms A and B in Adult and Fetal Human Tissues, and a Potentially Pathological Variant Affecting Only Isoform A in Two Patients with Cornelia de Lange Syndrome
}

\author{
Beatriz Puisac ${ }^{1}$, María-Esperanza Teresa-Rodrigo ${ }^{1}$, María Hernández-Marcos ${ }^{1}$, \\ Carolina Baquero-Montoya ${ }^{2}$, María-Concepción Gil-Rodríguez ${ }^{1}$, Torkild Visnes ${ }^{3}$, \\ Christopher Bot ${ }^{3}$, Paulino Gómez-Puertas ${ }^{4}$, Frank J. Kaiser ${ }^{5}$, Feliciano J. Ramos ${ }^{1,6}$, \\ Lena Ström ${ }^{3}$ and Juan Pié ${ }^{1, *}$ \\ 1 Unit of Clinical Genetics and Functional Genomics, Department of Pharmacology-Physiology and \\ Paediatrics, School of Medicine, University of Zaragoza, CIBERER-GCV02 and ISS-Aragon, \\ E-50009 Zaragoza, Spain; puisac@unizar.es (B.P.); eteresa@unizar.es (M.-E.T.-R.); \\ mhmarcos@unizar.es (M.H.-M.); mcgil@unizar.es (M.-C.G.-R.); framos@unizar.es (F.J.R.) \\ 2 Department of Pediatrics, Hospital Pablo Tobón Uribe, 05001000 Medellín, Colombia; carobaque@gmail.com \\ 3 Department of Cell and Molecular Biology, Karolinska Institute, SE-17177 Stockholm, Sweden; \\ Torkild.Visnes@ki.se (T.V.); Christopher.Bot@ki.se (C.B.); Lena.Strom@ki.se (L.S.) \\ 4 Molecular Modelling Group, Center of Molecular Biology “Severo Ochoa” (CSIC-UAM), Cantoblanco, \\ E-28049 Madrid, Spain; pagomez@cbm.csic.es \\ 5 Section for Functional Genetics at the Institute of Human Genetics, University of Lübeck, D-23538 Lübeck, \\ Germany; frank.kaiser@uk-sh.de \\ 6 Unit of Clinical Genetics, Department of Paediatrics, Hospital Clínico Universitario “Lozano Blesa”, \\ CIBERER-GCV02 and ISS-Aragon, E-50009 Zaragoza, Spain \\ * Correspondence: juanpie@unizar.es; Tel.: +34-976-761-677; Fax: +34-976-761-700
}

Academic Editor: Akila Mayeda

Received: 31 December 2016; Accepted: 16 February 2017; Published: 23 February 2017

\begin{abstract}
Cornelia de Lange syndrome (CdLS) is a congenital developmental disorder characterized by craniofacial dysmorphia, growth retardation, limb malformations, and intellectual disability. Approximately $60 \%$ of patients with CdLS carry a recognizable pathological variant in the NIPBL gene, of which two isoforms, A and B, have been identified, and which only differ in the C-terminal segment. In this work, we describe the distribution pattern of the isoforms A and B mRNAs in tissues of adult and fetal origin, by qPCR (quantitative polymerase chain reaction). Our results show a higher gene expression of the isoform $\mathrm{A}$, even though both seem to have the same tissue distribution. Interestingly, the expression in fetal tissues is higher than that of adults, especially in brain and skeletal muscle. Curiously, the study of fibroblasts of two siblings with a mild CdLS phenotype and a pathological variant specific of the isoform A of NIPBL (c.8387A > G; P.Tyr2796Cys), showed a similar reduction in both isoforms, and a normal sensitivity to DNA damage. Overall, these results suggest that the position of the pathological variant at the $3^{\prime}$ end of the NIPBL gene affecting only isoform $\mathrm{A}$, is likely to be the cause of the atypical mild phenotype of the two brothers.
\end{abstract}

Keywords: Cornelia de Lange syndrome; NIPBL isoform A; NIPBL isoform B; splicing variants; mRNA; adult tissues; fetal tissues; NIPBL pathological variant

\section{Introduction}

Brachmann-de Lange syndrome (BDLS) or Cornelia de Lange syndrome (CdLS, OMIM: 122,470, $300,590,610,759,614,701,300,882)$ is a rare congenital dysmorphogenic disorder with a high degree of 
variability in its clinical presentation [1]. The main characteristics are typical craniofacial dysmorphia, growth retardation, upper limb malformations, intellectual disability, hirsutism, and malformation of major organ systems, especially the digestive one [2].

In addition, CdLS shows a very extensive genetic heterogeneity, and pathological variants in NIPBL (5p13.1), SMC1A (Xp11.2), SMC3 (10q25), RAD21 (8q24), and HDAC8 (Xq13.1) genes, have been described [3-8]. All of these genes code for regulatory or structural proteins of the cohesin complex, essential for the proper function of several biological processes, such as chromosome segregation, DNA repair, gene expression regulation, and chromatin remodeling, among others [9]. Pathological variants in the cohesin regulator NIPBL are present in approximately $60 \%$ of classical CdLS patients $[3,4]$. In general, more damaging pathological variants (nonsense, frameshift) cause a more severe phenotype than less damaging pathological variants (missense) [10]. The NIPBL gene contains 47 exons and, although several transcripts have been identified in peripheral blood leukocytes [11], the main and largest size splicing variants are isoforms $\mathrm{A}$ and $\mathrm{B}$. Both isoforms are conserved in vertebrates and have identical aminoacids from 1 to 2683, while the C-terminal ends are different [4]. In the isoform A, exons 2-47 encode for the complete protein that has 2804 aminoacids. The alternative, isoform B, does not include exon 47 and ends in an expanded variant of exon 46, with a total of 2697 aminoacids.

Although previous studies have analyzed the total expression of the NIPBL gene by Northern blot [4], this paper reports, for the first time, on the mRNA distribution pattern of the A and B isoforms in different tissues of adult and fetal origin, quantified by quantitative PCR (qPCR). Moreover, we studied two brothers with CdLS and a missense pathological variant that exclusively affected the isoform A of the NIPBL gene. In addition, the presence of somatic mosaicism was ruled out and the effect of ionizing radiation on fibroblasts was assessed. Our results showed that these patients have a mild phenotype with less sensitivity to DNA damage, when compared to patients with severe CdLS, caused by mutations affecting both isoforms A and B.

\section{Results}

\subsection{Distribution of mRNA NIPBL Isoforms $A$ and B in Foetal and Adult Human Tissues}

In a first approximation by RT-PCR, the specific amplification of both isoforms A and B from the cDNA of different human tissues showed a similar tissue distribution pattern, although the levels of isoform A were always higher than those of B (Figure 1b). Both isoforms appeared to be expressed in a higher quantity and more homogeneously in fetal tissues, than in adult tissues.

\subsection{Quantitative mRNA Analysis of Isoforms A and B in Foetal and Adult Human Tissues}

The mRNA levels of the NIPBL isoforms were accurately quantified by qPCR, using specific primers for each of them (Figure 1a,c). For both isoforms $A$ and $B$, the value of 1 was assigned to the reference tissue of minimal expression, i.e., the adult skeletal muscle. The expression in all other tissues (adult and fetal) was then expressed in relation to this reference value.

The expression levels of isoform A in fetal tissues were higher than those in adult tissue, except for the liver, where the adult expression was higher ( 54 vs. 24 times higher than the reference level) (Figure 1c). The mRNA levels of isoform A in the fetal skeletal muscle and brain were 38 and 35 times higher than those in adult tissues, respectively (Figure 1c).

The highest expression of isoform A was obtained in the fetal kidney and adult pancreas (80 times higher than the reference tissue). Significant levels, in the range of 72-54 times higher than the reference, were found in the fetal thymus, spleen, lung, and heart, and the adult liver (Figure 1c). 
a 5

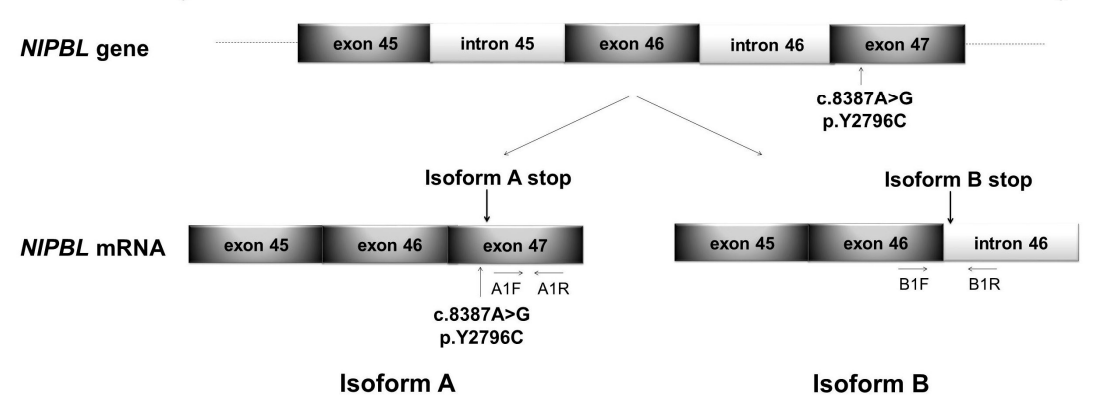

b

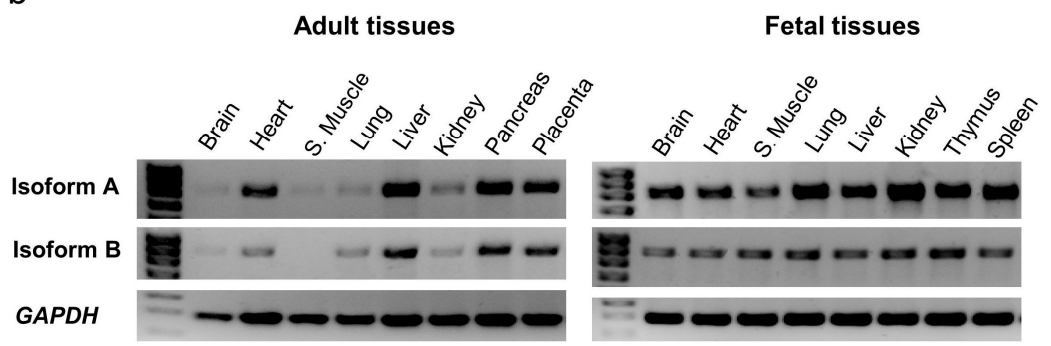

C

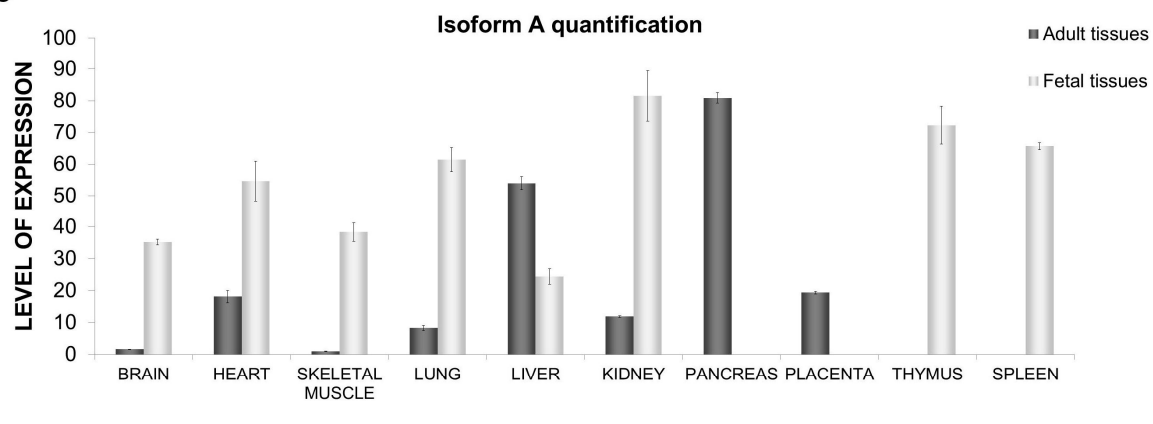

Isoform B quantification

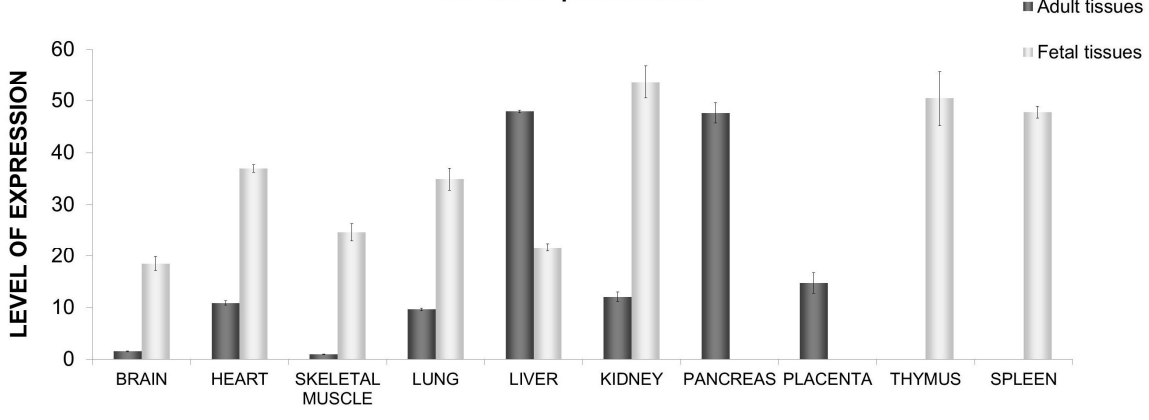

Figure 1. Study of NIPBL isoforms A and B in human tissues (a) Schematic representation of the $3^{\prime}$ region of the NIPBL gene and the resulting mRNA NIPBL isoforms A and B. The location of primers used for qPCR (quantitative polymerase chain reaction) to detect specific NIPBL isoforms A (AF1/AR1) and B (BF1/BR1) are indicated by arrows. The position of the pathological variant in exon 47, exclusive to isoform A, is also indicated; (b) RT-PCR (reverse transcription polymerase chain reaction) analysis of mRNA expression of NIPBL isoforms A and B. cDNA from normal adult and fetal tissues was used for the specific amplification of NIPBL isoforms A or B. Samples were analyzed by agarose gel-electrophoresis and GAPDH was used as a loading control; (c) Quantitative PCR analysis of mRNAs expression levels of NIPBL isoforms $\mathrm{A}$ and $\mathrm{B}$ in different tissues. $\mathrm{Ct}$ values were compared to standardized curves and normalized against GAPDH. The mean level of expression in adult skeletal muscle tissue was considered as 1 . All the tissues (adult and fetal) are expressed as a relative fold. Results are presented as means + standard deviation, $n=3$. 
The mRNA levels of isoform B, although clearly lower than that of isoform A, follow the same pattern of distribution (Figure 1c), as in Figure 1b. mRNA levels were higher in fetal tissues than in those of adults, except for the liver (47 times in adult/21 times in fetal), whereas in the fetal skeletal muscle and brain, they were 24 and 18 times higher than in adult tissue, respectively (Figure 1c). Peak levels were found in the fetal kidney, spleen, and thymus, and the adult pancreas and liver (53-47-fold), and minimum levels were found in adult skeletal muscle and the brain (Figure 1c).

\subsection{Structure Prediction of the C-Terminal Segment of the Isoforms A and B of the NIPBL Gene}

DP-Bind server (http://lcg.rit.albany.edu/dp-bind), a system for the sequence-based prediction of DNA-binding residues in proteins, suggested that the C-terminal segment of NIPBL isoform A (residues 2653-2804) is likely to be involved in DNA binding. A RaptorX system [12] was used to obtain a putative structure for this segment. The system proposed five different templates for the C-terminal residues 2541-2804 of isoform A, all of which had medium to medium-low internal scores for confidence. The structure with the better scores $\left(p\right.$-value $=1.4 \times 10^{-2}$, Score $=55, \mathrm{uGDT} / \mathrm{GDT}=27 / 16$ and uSeqID/SeqID = 16/9) corresponded to the DNA-binding domain of human cytomegalovirus DNA polymerase (Protein Data Bank entry $=3 \mathrm{kd} 1 \mathrm{E}$ ). Using this structure as a template, a 3D model was generated for the C-terminal domain of NIPBL isoform A (Figure S1). In brief, and according to this model, the aminoacidic sequence present in isoform A and absent in isoform B (residues 2683-2804), is predicted to form the domain that directly interacts with the putative DNA molecule, exhibiting a positive charge in the protein surface that is complementary to the negative charge of DNA. Conversely, NIPBL isoform B lacks this DNA-binding domain.

\subsection{Clinical Description}

The patients studied here are two stepbrothers of Spanish origin. The mother had a phenotype suggestive of mild CdLS. The mother had withdrawn the guardianship of her children and did not give her consent for the genetic study of herself. For this reason, she could not be included in the results.

\subsubsection{Patient 1}

Patient 1 is a 14-year-old male with a clinical diagnosis of CdLS, whose anthropometric data at birth and at his last evaluation are shown in Table 1. He had microcephaly, a low hair implantation line, arched eyebrows, synophrys, long and thin eyelashes, an atypical wide nose with a broad nasal bridge and tip, a long philtrum, a fine upper lip, a high palate, and microretrognatia (Figure 2a). He had normal hearing and no ophthalmological, gastrointestinal, or cardiac anomalies. Furthermore, he had a history of several low respiratory tract infections. He had pectum excavatum, scoliosis, flat feet, bilateral brachyclinodactyly of the fifth finger, bilateral 2-3 toe syndactyly, and 4th toe brachydactyly (Figure 2a, Table 1). Psychometric assessment showed a mild intellectual disability (IQ 44 in WISC-IV) and behavioral problems ADHD (attention deficit hyperactivity disorder), obsessive-compulsive behavior, aggressiveness, and self-injurious behavior) that needed pharmacological treatment. All together, and according to the severity classification criteria established by Kline et al. [2], findings in this patient fit with a mild CdLS spectrum.

\subsubsection{Patient 2}

Patient 2 is a 10-year-old male, the stepbrother of patient 1 , and the third child of his sibship. He had brachymicrocephaly, a shortened biparietal distance, arched eyebrows with synophrys, long and thin eyelashes, maxillar flattening, a wide nose with a broad nasal bridge, low-set and protuberant ears, micrognathia, and a short neck (Figure 2a, Table 1). He had 2-3 syndactyly in both feet (Figure 2a, Table 1). He also had strabismus of the left eye and a heart right bundle branch block with normal cardiac function. He had asthma until the age of three years. He had swallowing problems during the neonatal period, followed by recurrent vomiting and epigastralgia later in life, leading to the suspicion of gastroesophageal reflux disease (GERD) (Table 1). Psychiatric assessment showed a mild intellectual 
disability (IQ 75 with Raven test) and several behavioral problems (ADHD and self-injurious behavior) that needed pharmacological treatment (Table 1). As seen for his stepbrother, and according to the severity classification criteria established by Kline et al. [2], findings in this patient fit with a mild CdLS spectrum.

Table 1. Clinical features of the two patients.

\begin{tabular}{|c|c|c|c|}
\hline Clinical Data & & Patient 1 & Patient 2 \\
\hline \multirow{5}{*}{$\begin{array}{l}\text { Anthropometric data } \\
\text { (newborn) }\end{array}$} & Gestational age & 36 weeks & 35 weeks \\
\hline & Birth weight (g) & $2600(\mathrm{P} 10-25)$ & 2420 (P50) \\
\hline & Birth length $(\mathrm{cm})$ & $46(\mathrm{P} 10-25)$ & 46.7 (P50) \\
\hline & Birth OFC $(\mathrm{cm})$ & $31(\mathrm{P} 10-25)$ & $30.5(\mathrm{P} 10-25)$ \\
\hline & Intrauterine growth retardation & No & No \\
\hline \multirow{5}{*}{$\begin{array}{l}\text { Anthropometric data } \\
\text { (last evaluation) }\end{array}$} & Age at evaluation & 14 years 3 month & 10 years 8 month \\
\hline & Weight (kg) & $54(\mathrm{P} 25-50)$ & $36.3(\mathrm{P} 25-50)$ \\
\hline & Length $(\mathrm{cm})$ & $159.5(\mathrm{P} 25-50)$ & $136.5(\mathrm{P} 3-25)$ \\
\hline & OFC $(\mathrm{cm})$ & $52.5(\mathrm{P} 3-25)$ & $52.5(\mathrm{P} 25-50)$ \\
\hline & Postnatal growth retardation & No & No \\
\hline \multicolumn{2}{|l|}{ Limb abnomalities } & $\begin{array}{c}\text { Bilateral } \\
\text { brachydactyly—clinodactyly } \\
\text { 5th finger Bilateral 2-3 } \\
\text { syndactyly (feet) }\end{array}$ & $\begin{array}{c}2-3 \text { partial } \\
\text { syndactyly (feet) }\end{array}$ \\
\hline \multicolumn{2}{|l|}{ Developmental delay } & + & + \\
\hline \multicolumn{2}{|l|}{ Intellectual disability } & + & + \\
\hline \multicolumn{2}{|l|}{ Microcephaly } & + & - \\
\hline \multicolumn{2}{|c|}{ Behavior problems } & + & + \\
\hline \multicolumn{2}{|c|}{ Atypical wide nose with broad nasal bridge } & + & + \\
\hline \multicolumn{2}{|c|}{ Hypoacusia } & - & - \\
\hline \multirow{2}{*}{\multicolumn{2}{|c|}{$\begin{array}{l}\text { Gastroesophageal reflux disease } \\
\text { Feeding (swallowing) problems }\end{array}$}} & - & + \\
\hline & & - & + \\
\hline \multicolumn{2}{|c|}{ Hirsutism } & + & - \\
\hline \multicolumn{2}{|l|}{ Cutis marmorata } & - & - \\
\hline
\end{tabular}

OFC: Occipito-frontal circumference; + present/- not present.

\subsection{Genetic Diagnosis and Pyrosequencing Analysis in Different Tissues}

The pathological variant identified in the blood DNA of both patients was c.8387A > G, p.Tyr2796Cys, located at the last exon (E47) of the NIPBL gene. This missense pathological variant causes the change of aminoacid Tyrosine 2796 to Cysteine, and it only affects isoform A (Figure 1a). The residue (Y2796) is highly conserved in vertebrates. The Polyphen-2 program [13] predicted a probably damaging effect of this missense pathological variant on the NIPBL protein, with a score of 0.999. The SNPpredict program [14] also considers this pathological variant as deleterious, with an expected accuracy of $87 \%$. The presence of other variants in the NIPBL gene, or a mutation in the other causal genes of the syndrome $S M C 1 A, S M C 3, H D A C 8$, and RAD21, have been ruled out by sequencing.

On the other hand, pyrosequencing was used for the precise quantification of the wild type and mutant alleles in the genomic DNA of the different tissues (leukocytes, fibroblasts, buccal epithelial cells, and epithelial cells from the urine). In all of the tissues, the expression of one allele against the other was equivalent, with values which were consistently around $50 \%$ (Figure $2 \mathrm{~b}$ ). 
a

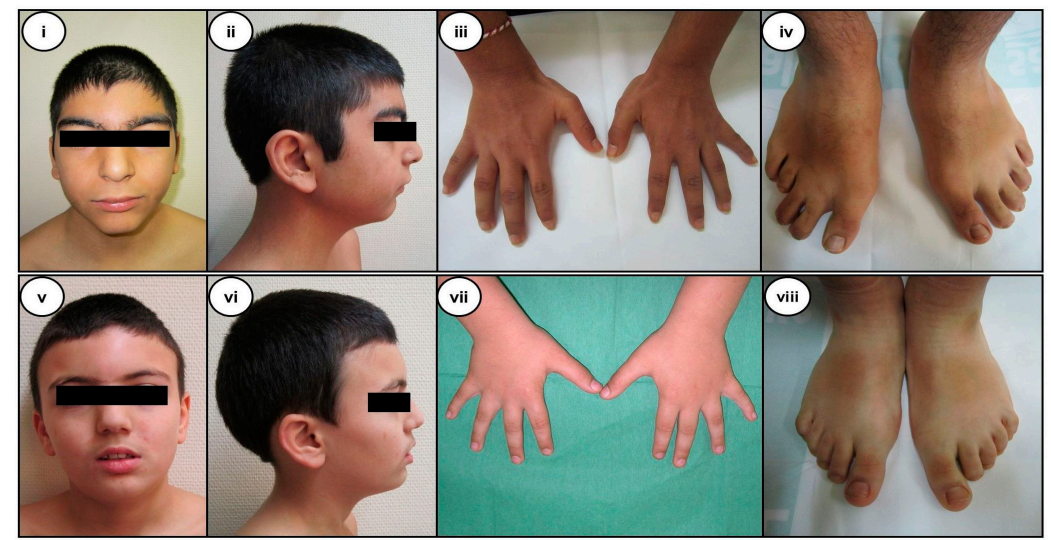

b

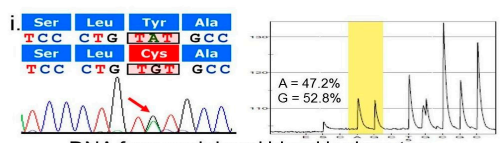

DNA from peripheral blood leukocytes
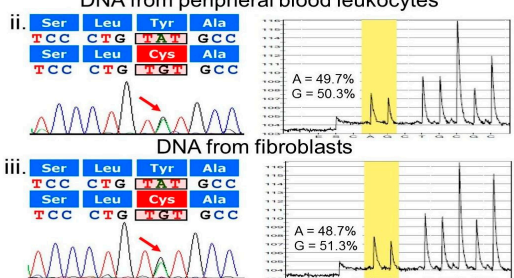

DNA from mucosa epithelial cells

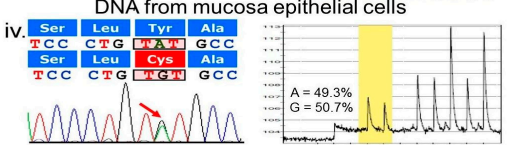

DNA from urine epithelial cells

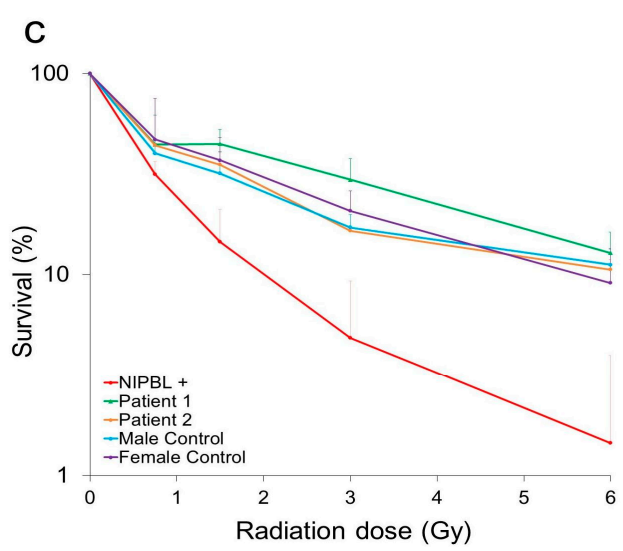

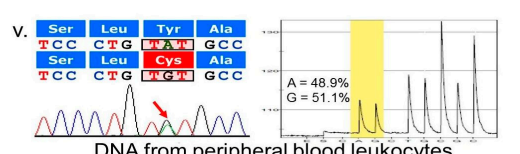

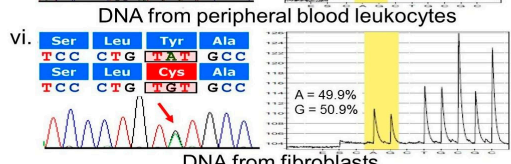

DNA from fibroblasts

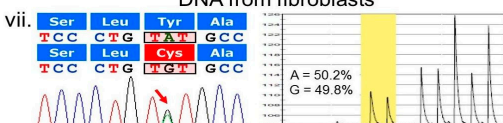

DNA from mucosa epithelial cells

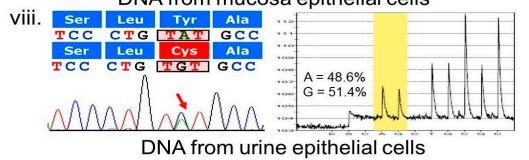

d
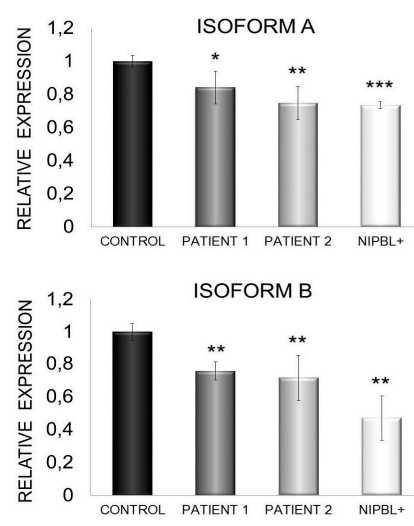

Figure 2. Overview of the phenotype and molecular findings of the two patients (a) Phenotype of patient 1 and patient 2. Frontal and lateral view of each patient (i, v, ii, vi), hands (iii, vii) and feet (iv, viii); (b) Detection of the pathological variant c.8387A > G (p.Tyr2796Cys) in patient 1 and patient 2 on DNA from peripheral blood leukocytes (i-v), fibroblasts (ii-vi), epithelial cells from oral mucosa (iii-vii), and from urinary tract (iv-viii). For each tissue, the chromatogram corresponding to Sanger sequencing and pyrosequencing quantification are shown; the red arrows indicate the nucleotide substitution position. In pyrograms, $A$ versus $G$ peak are yellow shading (c) Evaluation of the sensitivity to radiation referred to as a percentage of surviving cells vs. radiation dose. Each experiment was performed at least twice in triplicate. Note the similar pattern shown for patients $\mathrm{P} 1$ and $\mathrm{P} 2$, and healthy controls, and the different pattern shown for a NIPBL+ patient; (d) Quantitative PCR analysis of mRNA's expression levels of NIPBL isoforms A and B from fibroblasts of patients and a control ${ }^{*} p<0.05,{ }^{* *} p<0.01,{ }^{* * *} p<0.001$. 


\subsection{DNA Damage Sensibility in Patients' Fibroblasts}

A study of the sensitivity to DNA damage was carried out by exposure to increasing doses of gamma radiation to the fibroblasts of the two patients, two healthy control subjects, and one patient with a pathological variant in NIPBL, affecting both isoforms $\mathrm{A}$ and $\mathrm{B}$, as previously reported $[15,16]$. The survival of cells was monitored using a colony formation assay. Although there was some variation between the results, patient 1 and patient 2 were no more sensitive to ionizing radiation than the healthy child control cells $(p>0.05)$. In contrast, the fibroblasts from the patient with NIPBL+ was easily sensitized by ionizing radiation, even at very low radiation doses (Figure 2c).

\subsection{Quantification of mRNA NIPBL Isoforms $A$ and B in Patients' Fibroblasts}

The mRNA levels of NIPBL isoforms A and B were then assessed for the fibroblasts of patient 1 , patient 2, and the same NIPBL+ patient in Figure $2 c$, using $\mathrm{qPCR}$ with the primers and strategy described for normal tissues. For each of the isoforms, a 100\% value was assigned to the mean expression obtained in fibroblasts from control individuals, and the expression in each patient was reported as a percentage relative to that value. In the patients studied, reduced levels of isoform $\mathrm{A}$ were observed when compared to controls: patient $1(84 \%, p<0.05)$, patient $2(74.6 \%, p<0.01)$, and the NIPBL+ patient. $(73.4 \%, p<0.001)$ Isoform $B$ was also reduced in all patients, with the following values: patient $1(75.5 \%, p<0.01)$, patient $2(71.5 \%, p<0.01)$, and the NIPBL+ patient $(47 \%, p<0.01)$ (Figure 2d).

\section{Discussion}

Heterozygous pathological variants in the NIPBL gene are the main cause of Cornelia de Lange syndrome, the most frequent cohesinopathy. In order to better understand the pathogenesis of the syndrome, it is necessary to study the functions of the NIPBL protein in greater depth, implicated not only in cohesin loading, but also in the regulation of gene expression during development $[17,18]$. This variety of functions could be achieved through the generation of alternative transcripts by splicing, one of the most efficient mechanisms of protein diversification [19]. For this reason, we studied the two main splice variants of the NIPBL gene, isoforms $A$ and $B$, in adult and fetal human tissues, and in two patients with CdLS who were harbouring a pathological variant specific for isoform A. Isoforms A and B differ only at the carboxy-terminal end, and thus, isoform A encodes the complete protein, whereas isoform B lacks exon 47 and ends in an expanded form of exon 46 [4]. Although the function of the final segment that differentiates them is unknown, it has been suggested that it interacts with DNA [20]. Our in silico analyses seem to confirm this hypothesis and suggest that the specific segment of isoform A interacts with DNA (Figure S1). However, further studies will be needed to confirm this hypothesis.

Results of qPCR showed that isoform A is present in higher amounts than isoform B, in all the studied tissues. Despite the differences, both variants have an extraordinarily similar pattern of tissue expression (Figure 1b,c), which seems to support a coordinated regulatory mechanism, as has been described for other genes [21,22]. On the other hand, the analysis of the number of transcripts reflects a higher production in fetal tissues, which could be justified by the importance of the cohesins for the regulation of gene expression during embryonic development [9]. Interestingly, brain and skeletal muscle had a very low expression in adult tissues, and this was up to 35 and 38 times lower than in fetal tissues, respectively. Liver tissue is, however, the exception to the rule, with a lower expression in fetal tissue, than in adult tissue. Among all of the tissues analyzed, the highest expression was found in the kidney, thymus, spleen, and fetal lung, as well as in the pancreas and adult liver. These values broadly coincide with the semi-quantitative total expression obtained by Tonkin [4] using Nothern blot, although he obtained lower values for an adult liver. However, there is no apparent direct relationship between the tissues with a higher expression of NIPBL and the clinical expressivity of the syndrome, since CdLS patients, in general, do not have a severe involvement of the kidney, liver, or pancreas. 
However, a better understanding of the expression of these isoforms in specific tissues could be a first step in the search for possible therapeutic options for CdLS, based on the modulation of splicing.

Here, we also report on two stepbrothers with a new missense c.8387A > G pathological variant (Tyr2796Cys) located in exon 47, which only affects the isoform A of the NIPBL gene. Both patients have a mild phenotype according to the Kline's severity score. Both show some characteristic facial features of CdLS, but also have a wide nose with a broad nasal bridge and tip, similar to that described in patients who have CdLS with pathological variants in the HDAC8 gene [23]. The last evaluation at 14 years of patient 1 , and at 10 years of patient 2 , showed a normal growth development and a mild intellectual disability that reinforces the initial diagnosis of low affectation. In addition, somatic mosaicism was ruled out as the possible cause of the mild phenotype. Studies on tissues of different embryological origin: leukocytes and fibroblasts (mesoderm), oral mucosa (ectoderm), and urinary epithelium (endoderm and ectoderm), showed a percentage of mutated alleles close to $50 \%$ (Figure 2b).

Despite its limited clinical information, a study of other pathological variants exclusive to isoform A, described in the LSDB database [24], revealed a tendency to produce mild phenotypes, even in frameshift variants (c.8377C > T, p.Arg2793Term 2; c.8364delT, ex47 p.Val2789PhefsX36), similar to the findings observed in our patients.

It also appears that the mild clinical manifestations of the two stepbrothers correspond to what is observed at the cellular level. Although it is well known that CdLS patients' cells have an increased sensitivity to DNA damage [16,25], our patients' fibroblasts behaved the same as controls, i.e., they were significantly less affected by ionizing radiation than a CdLS patient with a pathological variant affecting both isoforms (Figure 2c). This is the first report of patients with a clinical phenotype of CdLS and a mutation in NIPBL that do not show sensitivity to DNA damage. It is also possible that the phenotype in these patients was dependent on a quantitatively different expression of both isoforms, since isoform B is not altered by the pathological variant. However, a decrease in isoform A ( $16 \%$ and $25.4 \%$ comparing to the control) was observed in both patients, similar to what was observed in isoform B (24.5\% and $28.5 \%$ ) (Figure $2 \mathrm{~d}$ ). These findings suggest that the reduction of expression occurs before mRNA maturation. In the future, quantification of the expression of these isoforms in a large number of patients with distinct pathological variants in NIPBL, may be essential for understanding the contribution of each isoform in the phenotype of CdLS patients.

In conclusion, our results indicate that isoform A is more expressed than isoform B, although they display a very similar tissue distribution pattern. In addition, both isoforms are more abundant in foetal tissues than in adult tissues, especially in brain and skeletal muscle, which can be related to the development of these organs. It is also suggested that patients who only have a pathological variant in isoform A, have a milder CdLS clinical phenotype and a lower sensitivity to DNA damage.

\section{Materials and Methods}

\subsection{Identification of NIPBL Isoforms $A$ and B in Foetal and Adult Human Tissues}

cDNA from different foetal and adult human tissues: brain, heart, skeletal muscle, lung, liver, kidney, pancreas (adult), placenta (adult), thymus (foetal), and spleen (foetal), were purchased from Clontech (Mountain View, CA, USA). Using the primers sF43 (5'-ACATTACACTC TCAGTTTCTG-3'), sR47 (5'-ACGTCAACACTTTCATTC-3'), and sR46b (5'-GATAGAGATTCTCTACTTACCCG-3'), we specifically amplified isoform A (sF43/SR47) and isoform B (sF43/SR46b). The identity of all PCR products was confirmed by DNA sequencing, using the "ABI Prism BigDye Terminator Cycle Sequencing v2.0" Kit (Applied Biosystems, Foster City, CA, USA), and was analyzed on an Applied Biosystems 5700 DNA sequencer (Foster City, CA, USA).

\subsection{Quantification of $m R N A$ NIPBL Isoforms $A$ and $B$ by $q P C R$ in Human Tissues}

The quantification of NIPBL isoforms A and B was performed for the cDNA of foetal and adult human tissues from normalized commercial panels Human Fetal MTC Panel and Human MTC Panel I, 
respectively (Clontech Laboratories, Mountain View, CA, USA). Isoform A was amplified using primers NIPBL A1F (5'-GGCAGCACAGATGAATGAAAG-3') and NIPBL A1R (5'-CTTGCAATTT GTGGTCGATCTT- $\left.3^{\prime}\right)$, and isoform B with primers NIPBL B1F (5'-AATACAGCAGCAGAGACAG AAG-3') and NIPBL B1R (5'-CGAAATACTTGACTCCTCCTC-3'). Standardized curves were used to quantify the expression of each isoform. To create the standardized curves, the cDNA of NIPBL isoforms A and B were inserted into the $\mathrm{pCR}-2.1-\mathrm{TOPO}$ vector, according to the manufacturer's protocol. Plasmids were quantified by spectrophotometric analysis at $260 \mathrm{~nm}$, and standardized curves were based on a 10-fold serial dilution of the different cloned isoforms. The $C_{\mathrm{t}}$ (Cycle threshold) values of the samples were interpolated to the corresponding standardized curve. Reactions were performed by using a Power SYBR Green Master Mix, following the manufacturer's recommendations. Each reaction contained $1 \mu \mathrm{L}$ of cDNA in a $20 \mu \mathrm{L}$ mixture. In each experiment, control samples were included: (1) a no template control (NTC); and (2) a no reverse transcriptase (-RT) control. The identity of the PCR products and their purity in each sample were controlled after the last amplification cycle by melting curve analysis. The same procedure was used on GAPDH, in order to normalize it. All of the samples were quantified in triplicate, and the average values were calculated.

\subsection{Patients}

This study includes two new Spanish patients who met the criteria for CdLS, according to Kline et al. [2]. Ethical recommendations of the Declaration of Helsinki were followed. The patients' tutor signed the informed consent to participate in the study. To establish comparisons, we also used cells from two anonymous unaffected individuals and from a patient with CdLS, previously reported by us $[15,16]$. The present study was approved by the Ethics Committee for Clinical Research of Aragon, on February 2013 (reference number: 04/2013).

\subsection{Genetic Diagnosis and Pyrosequencing Analysis}

We collected DNA samples from both patients from four tissues with similar and/or of different embryological origin (mesoderm (peripheral blood leukocytes and fibroblasts), ectoderm (oral mucosa epithelial cells), and endoderm/ectoderm (urinary epithelium), by standardized protocols. The coding regions and flanking intronic sequences of NIPBL (exons 2-47), SMC1A, SMC3, HDAC8, and RAD21 were screened for pathological variants by PCR amplification of blood genomic DNA. The PCR products were purified with USB ExoSAP-IT PCR Product Cleanup (Affymetrix, Santa Clara, CA, USA), following the manufacturer's instructions, and were sequenced in a ADN3130 Genetic Analyzer (Applied Biosystems). Parental genotypes were screened to assess whether the variant was de novo or inherited. NIPBL cDNA was numbered according to the NIPBL isoform 1 (GenBank accession number NM 000642). The pathological variant nomenclature was applied following the instructions from the Human Genome Variation Society [26].

Quantification of the relative amount of each allele in DNA samples from blood leukocytes, fibroblasts, oral mucosa epithelial cells, and urinary epithelium, were performed by the pyrosequencing analysis of biotinylated PCR products. They were performed in triplicate using the PSQ 96MA pyrosequencer (Qiagen, Hilden, Germany), following the manufacturer's instructions (primers available on request). The level of heterozygosity of each sample was calculated by PSQ 96TMMA allele quantification software (Qiagen), and validated by Sanger sequencing.

\subsection{Structural In Silico Modeling of C-Terminal Domain of NIPBL Isoforms A and B and Bioinformatic Analysis of the Missense Pathological Variant in Isoform A}

Structural in silico analysis of the C-terminal domain of NIPBL isoforms A (residues 2541-2804) and B (residues 2541-2697) was performed by using the protein structure prediction system "RaptorX" [12]. From the five putative templates suggested by the system, the one with the best scoring values ( $p$-value, Score, uGDT and GDT, uSeqID, and SeqID) was selected and used to build a 3D structure model for the protein C-terminal domain of isoform A. 
The pathogenicity of the missense pathological variant was tested with Poly-Phen-2 [13] and PredictSNP [14].

\subsection{Cell Culture and Colony Formation Assay}

Fibroblast cell lines were maintained in a 1:1 mix of RPMI-1640 (R8758, Sigma-Aldrich, Saint Louis, MO, USA) and Ham's F-10 nutrient mixture (N6908, Sigma-Aldrich), supplemented with 10\% FBS (F7524, Sigma-Aldrich), 1:100 dilutions of sodium pyruvate (11360, Gibco, Carlsbad, CA, USA) and 1:200 dilution of penicillin-streptomycin (P4333, Sigma-Aldrich). Cells were maintained for less than 12 passages at $37^{\circ} \mathrm{C}$ and $5 \% \mathrm{CO}_{2}$.

For colony formation assays, cells were seeded in 6-well dishes at the following dilutions: 125, 250, 500, 1000, 2000, and 4000 cells/well. Cells were allowed to attach for $1-3 \mathrm{~h}$ and were then irradiated with the indicated doses, using a 137Cs radiation source (IBL 677, CIS Bio international Bedford, MA, USA). Following irradiation, cells were allowed to grow for 12-17 days, washed with PBS, fixed in methanol for 6-9 min, air dried, and stained with a filtered 1:20 dilution of the Giemsa stain (GS500, Sigma-Aldrich).

\subsection{Quantification of $m R N A$ from NIPBL Isoforms $A$ and B in Patients' Fibroblasts}

The total RNA from the fibroblasts of patients and controls was extracted using the PAXgene Blood RNA Kit (PreAnalaytiX, Hombrechtikon, Switzerland), according to the manufacturer's instructions. Single-stranded cDNAs were synthesized from 500 ng of RNA, using the First Strand Synthesis Kit (Thermo Fisher, Boston, MA, USA) with random hexamers.

qPCR was performed to quantify both isoforms with the same primers and conditions described in the quantification of tissues. The same procedure was used on GAPDH for normalization. The patients' results were compared to a normal healthy control. The data were log transformed, which made the data normally distributed, and a Student's $t$ test was performed to compare gene expression data. Differences between experimental groups were considered significant when $p<0.05$.

Supplementary Materials: Supplementary materials can be found at www.mdpi.com/1422-0067/18/3/481/s1.

Acknowledgments: We sincerely thank the patients' family for participating in this study. This work was supported by: The Spanish Ministry of Health—Fondo de Investigación Sanitaria (FIS) (Ref: PI15/00707); the Diputación General de Aragón (Grupo Consolidado B20), European Social Fund ("Construyendo Europa desde Aragón") CHROMATIN-Net funded by the German Federal Ministry of Education and Research (BMBF) to Frank J. Kaiser. The Spanish Ministry of Economy (Refs: IPT2011-0964-900000 and SAF2011-13156-E) to Paulino Gómez-Puertas. Swedish Research Council to Lena Ström and the Norwegian Research Council (205217) to Torkild Visnes Beatriz Puisac, María Hernández-Marcos, María-Esperanza Teresa-Rodrigo, María-Concepción Gil-Rodríguez, Feliciano J. Ramos and Juan Pié are members of CIBERER-GCV02 and ISS-Aragon at the School of Medicine, University of Zaragoza and the Hospital Clínico Universitario "Lozano Blesa".

Author Contributions: Beatriz Puisac and Juan Pié initiated the physiological splicing studies. Feliciano J. Ramos and Carolina Baquero-Montoya identified, characterized, and provided patient data. María Hernández-Marcos and María-Concepción Gil-Rodríguez performed the genetic diagnosis. Lena Ström, Torkild Visnes, Christopher Bot, Beatriz Puisac, and María-Esperanza Teresa-Rodrigo performed the molecular and cellular studies. Paulino Gómez-Puertas performed the in silico structural analysis. María-Esperanza Teresa-Rodrigo and María Hernández-Marcos analysed statistical data and prepared the figures. Beatriz Puisac, Frank J. Kaiser, Feliciano J. Ramos, Lena Ström and Juan Pié drafted and reviewed the manuscript. All authors analysed data, discussed the results, and were provided with the opportunity to comment on the manuscript.

Conflicts of Interest: The authors declare no conflict of interest.

\section{References}

1. Opitz, J.M. The Brachmann-de Lange syndrome. Am. J. Med. Genet. 1985, 22, 89-102. [CrossRef] [PubMed]

2. Kline, A.D.; Krantz, I.D.; Sommer, A.; Kliewer, M.; Jackson, L.G.; FitzPatrick, D.R.; Levin, A.V.; Selicorni, A. Cornelia de Lange syndrome: Clinical review, diagnostic and scoring systems, and anticipatory guidance. Am. J. Med. Genet. A 2007, 143A, 1287-1296. [CrossRef] [PubMed] 
3. Krantz, I.D.; McCallum, J.; DeScipio, C.; Kaur, M.; Gillis, L.A.; Yaeger, D.; Jukofsky, L.; Wasserman, N.; Bottani, A.; Morris, C.A.; et al. Cornelia de Lange syndrome is caused by mutations in NIPBL, the human homolog of Drosophila melanogaster Nipped-B. Nat. Genet. 2004, 36, 631-635. [CrossRef] [PubMed]

4. Tonkin, E.T.; Wang, T.J.; Lisgo, S.; Bamshad, M.J.; Strachan, T. NIPBL, encoding a homolog of fungal Scc2-type sister chromatid cohesion proteins and fly Nipped-B, is mutated in Cornelia de Lange syndrome. Nat. Genet. 2004, 36, 636-641. [CrossRef] [PubMed]

5. Musio, A.; Selicorni, A.; Focarelli, M.L.; Gervasini, C.; Milani, D.; Russo, S.; Vezzoni, P.; Larizza, L. X-linked Cornelia de Lange syndrome owing to SMC1L1 mutations. Nat. Genet. 2006, 38, 528-530. [CrossRef] [PubMed]

6. Deardorff, M.A.; Kaur, M.; Yaeger, D.; Rampuria, A.; Korolev, S.; Pie, J.; Gil-Rodríguez, C.; Arnedo, M.; Loeys, B.; Kline, A.D.; et al. Mutations in cohesin complex members SMC3 and SMC1A cause a mild variant of Cornelia de Lange syndrome with predominant mental retardation. Am. J. Hum. Genet. 2007, 80, 485-494. [CrossRef] [PubMed]

7. Deardorff, M.A.; Wilde, J.J.; Albrecht, M.; Dickinson, E.; Tennstedt, S.; Braunholz, D.; Mönnich, M.; Yan, Y.; Xu, W.; Gil-Rodríguez, M.C.; et al. RAD21 mutations cause a human cohesinopathy. Am. J. Hum. Genet. 2012, 90, 1014-1027. [CrossRef] [PubMed]

8. Deardorff, M.A.; Bando, M.; Nakato, R.; Watrin, E.; Itoh, T.; Minamino, M.; Saitoh, K.; Komata, M.; Katou, Y.; Clark, D.; et al. HDAC8 mutations in Cornelia de Lange syndrome affect the cohesin acetylation cycle. Nature 2012, 489, 313-317. [CrossRef] [PubMed]

9. Remeseiro, S.; Losada, A. Cohesin, a chromatin engagement ring. Curr. Opin. Cell. Biol. 2013, $25,63-71$. [CrossRef] [PubMed]

10. Mannini, L.; Cucco, F.; Quarantotti, V.; Krantz, I.D.; Musio, A. Mutation spectrum and genotype-phenotype correlation in Cornelia de Lange syndrome. Hum. Mutat. 2013, 34, 1589-1596. [CrossRef] [PubMed]

11. Teresa-Rodrigo, M.E.; Eckhold, J.; Puisac, B.; Dalski, A.; Gil-Rodríguez, M.C.; Braunholz, D.; Baquero, C.; Hernández-Marcos, M.; de Karam, J.C.; Ciero, M.; et al. Functional characterization of NIPBL physiological splice variants and eight splicing mutations in patients with Cornelia de Lange syndrome. Int. J. Mol. Sci. 2014, 15, 10350-10364. [PubMed]

12. Källberg, M.; Haipeng, W.; Sheng, W.; Jian, P.; Zhiyong, W.; Hui, L.; Jinbo, X. Template-based protein structure modeling using the RaptorX web server. Nat. Protoc. 2012, 7, 1511-1522. [CrossRef] [PubMed]

13. PolyPhen-2: Prediction of Functional Effects of Human nsSNPs. Available online: http://genetics.bwh. harvard.edu/pph/ (accessed on 21 December 2016).

14. PredictSNP: Consensus Classifiers for Prediction of Disease-Related Mutations. Available online: http:/ /loschmidt.chemi.muni.cz/predictsnp/ (accessed on 21 December 2016).

15. Pié, J.; Gil-Rodríguez, M.C.; Ciero, M.; López-Viñas, E.; Ribate, M.P.; Arnedo, M.; Deardorff, M.A.; Puisac, B.; Legarreta, J.; de Karam, J.C.; et al. Mutations and variants in the cohesion factor genes NIPBL, $S M C 1 A$, and SMC3 in a cohort of 30 unrelated patients with Cornelia de Lange syndrome. Am. J. Med. Gene A 2010, 152A, 924-929. [CrossRef] [PubMed]

16. Enervald, E.; Du, L.; Visnes, T.; Björkman, A.; Lindgren, E.; Wincent, J.; Borck, G.; Colleaux, L.; Cormier-Daire, V.; van Gent, D.C. A regulatory role for the cohesin loader NIPBL in nonhomologous end joining during immunoglobulin class switch recombination. J. Exp. Med. 2013, 210, 2503-2513. [CrossRef] [PubMed]

17. Dorsett, D. Cohesin, gene expression and development: Lessons from Drosophila. Chromosom. Res. 2009, 17, 185-200. [CrossRef] [PubMed]

18. Muto, A.; Ikeda, S.; Lopez-Burks, M.E.; Kikuchi, Y.; Calof, A.L.; Lander, A.D.; Schilling, T.F. NIPBL and mediator cooperatively regulate gene expression to control limb development. PLoS Genet. 2014, 10, e1004671. [CrossRef] [PubMed]

19. Nilsen, T.W.; Graveley, B.R. Expansion of the eukaryotic proteome by alternative splicing. Nature 2010, 463, 457-463. [CrossRef] [PubMed]

20. Yan, J.; Saifi, G.M.; Wierzba, T.H.; Withers, M.; Bien-Willner, G.A.; Limon, J.; Stankiewicz, P.; Lupski, J.R.; Wierzba, J. Mutational and genotype-phenotype correlation analyses in 28 Polish patients with Cornelia de Lange syndrome. Am. J. Med. Genet. A 2006, 140, 1531-1541. [CrossRef] [PubMed]

21. Holmberg, J.; Clarke, D.L.; Frisén, J. Regulation of repulsion versus adhesion by different splice forms of an EPH receptor. Nature 2000, 408, 203-206. [PubMed] 
22. Chen, T.; Tang, Z.; Yan, A.; Li, W.; Lin, H. Molecular cloning and mRNA expression analysis of two GH secretagogue receptor transcripts in orange-spotted grouper (Epinephelus coioides). J. Endocrinol. 2008, 199, 253-265. [CrossRef] [PubMed]

23. Kaiser, F.J.; Ansari, M.; Braunholz, D.; Gil-Rodriguez, M.C.; Decroos, C.; Wilde, J.J.; Fincher, C.T.; Kaur, M.; Bando, M.; Amor, D.J.; et al. Loss-of-function HDAC mutations cause a phenotypic spectrum of Cornelia de Lange syndrome-like features, ocular hypertelorism, large fontanelle and X-linked inheritance. Hum. Mol. Genet. 2014, 23, 2888-2900. [CrossRef]

24. LOVD—Leiden Open Variation Database. Available online: http://www.lovd.nl/3.0/home (accessed on 21 December 2016).

25. Vrouwe, M.G.; Elghalbzouri-Maghrani, E.; Meijers, M.; Schouten, P.; Godthelp, B.C.; Bhuiyan, Z.A.; Redeker, E.J.; Mannens, M.M.; Mullenders, L.H.; Pastink, A.; et al. Increased DNA damage sensitivity of Cornelia de Lange syndrome cells: Evidence for impaired recombinational repair. Hum. Mol. Genet. 2007, 16, 1478-1487. [CrossRef] [PubMed]

26. Human Genome Variation Society. Available online: http://www.hgvs.org/ (accessed on 21 December 2016).

(C) 2017 by the authors. Licensee MDPI, Basel, Switzerland. This article is an open access article distributed under the terms and conditions of the Creative Commons Attribution (CC BY) license (http:/ / creativecommons.org/licenses/by/4.0/). 